\title{
Visual cortex plasticity evokes excitatory alterations in the hippocampus
}

\author{
Marian Tsanov ${ }^{1,2}$ and Denise Manahan-Vaughan ${ }^{1,2 *}$ \\ 1 Department of Experimental Neurophysiology, Medical Faculty, Ruhr University Bochum, Bochum, Germany \\ 2 International Graduate School of Neuroscience, Ruhr University Bochum, Bochum, Germany
}

Edited by:

Sidarta Ribeiro, Edmond and Lily Safra International Institute of Neuroscience of Natal, Brazil; Federal University of Rio Grande do Norte, Brazil

\section{Reviewed by:}

Edward Tehovnik, Massachusetts Institute of Technology, USA Rodrigo N. Romcy-Pereira, Federal University of Espirito Santo, Brazil

\section{*Correspondence:}

Denise Manahan-Vaughan, Department of Experimental Neurophysiology, Medical Faculty, Ruhr University Bochum, MABF

01/551, Universitaetsstr. 150, 44780

Bochum, Germany.

e-mail:dmv-igsn@rub.de
The integration of episodic sequences in the hippocampus is believed to occur during theta rhythm episodes, when cortico-hippocampal dialog results in reconfiguration of neuronal assemblies. As the visual cortex (VC) is a major source of sensory information to the hippocampus, information processing in the cortex may affect hippocampal network oscillations, facilitating the induction of synaptic modifications. We investigated to what degree the field activity in the primary VC, elicited by sensory or electrical stimulation, correlates with hippocampal oscillatory and synaptic responsiveness, in freely behaving adult rats. We found that the spectral power of theta rhythm $(4-10 \mathrm{~Hz})$ in the dentate gyrus (DG), increases in parallel with high-frequency oscillations in layer $2 / 3$ of the $\mathrm{VC}$ and that this correlation depends on the degree of exploratory activity. When we mimic robust thalamocortical activity by thetaburst application to dorsal lateral geniculate nucleus, a hippocampal theta increase occurs, followed by a persistent potentiation of the DG granule field population spike. Furthermore, the potentiation of DG neuronal excitability tightly correlates with the concurrently occurring VC plasticity. The concurrent enhancement of VC and DG activity is also combined with a highly negative synchronization between hippocampal and cortical low-frequency oscillations. Exploration of familiar environment decreases the degree of this synchrony. Our data propose that novel visual information can induce high-power fluctuations in intrinsic excitability for both VC and hippocampus, potent enough to induce experience-dependent modulation of corticohippocampal connections. This interaction may comprise one of the endogenous triggers for long-term synaptic plasticity in the hippocampus.

Keywords: dentate gyrus, visual cortex, oscillations, in vivo

\section{INTRODUCTION}

The adult visual cortex (VC) possesses the remarkable ability to change its neuronal connectivity through experience (Sur et al., 2002). This remodeling of synapses involved in cortical activity is mediated by bidirectional changes in synaptic strength (Heynen et al., 2000; Liu et al., 2004) and these changes are related to structural plasticity through the loss and gain of synapses (Majewska et al., 2006). Experimental evidence derived from in vitro studies suggests that synaptic plasticity may be involved in information processing in the adult sensory cortex (Bear, 2003). Plasticity processes in the adult $\mathrm{VC}$ are also proposed to underlie different forms of visual perceptual learning (Hegde, 2006; Karmarkar and Dan, 2006). Recent data indicate that the adult VC serves as a synaptic network, where the direction of change in synaptic strength depends on the immediate visual experience (Tsanov and Manahan-Vaughan, 2007a,b). Thus, the VC dynamically modifies its ability to process visual stimuli, and therefore may affect the processing of sensory information in related brain areas (Abbott et al., 1997). Importantly, synaptic plasticity occurring in the adult primary VC may influence the ability of the hippocampus to integrate spatial episodes in time (Ji and Wilson, 2007). This interaction may comprise a key element in cortico-hippocampal transfer of information and the subsequent formation of the spatial memories.
The current work aimed to investigate the postulate that cortical synaptic plasticity influences information processing in the hippocampus, such that visual sensory information is integrated into episodic memory (Eichenbaum, 2000; Tsanov and Manahan-Vaughan, 2008). Therefore novel visual stimuli should be able to induce more robust alterations in the cortico-hippocampal axis, compared to already experienced ones. For memory to occur, plasticity modifications must be potent enough to evoke long-term change of synaptic weight (Castro et al., 1989; Barnes, 1995; Moser, 1995; Morris and Frey, 1997). In an effort to more closely approximate endogenous conditions for synaptic plasticity many researchers have studied the relationship between hippocampal long-term potentiation (LTP) and neuronal oscillations during exploratory behaviour. One of the more convincing links between learning and hippocampal LTP involves the use of theta-frequency stimulation, establishing a connection between theta rhythm and LTP (Larson et al., 1986; Rose and Dunwiddie, 1986; Buzsáki et al., 1987; Larson and Lynch, 1989). This rhythm is naturally prominent in the hippocampus during exploratory behaviour including representation of visuospatial information (O'Keefe and Nadel, 1978; O'Keefe and Recce, 1993; Skaggs et al., 1996; Kahana et al., 1999), REM sleep (Jouvet, 1969), active exploration (Vanderwolf, 1969), and memory formation and retrieval (Larson and Lynch, 1986; Buzsáki, 1989). Theta rhythm is 
believed to be critical for temporal coding/decoding of active neuronal ensembles and the modification of synaptic weights (Buzsáki, 2002). A major goal in our study therefore was to investigate the influence of behavioural and stimulation protocols of visual system on hippocampal theta oscillations. During theta behavioural states there is a prominent enhancement of another field oscillation - at gamma frequency $(40-100 \mathrm{~Hz})$. A recent study has suggested that temporal coordination of neocortical gamma oscillations by hippocampal theta is a mechanism by which information contained in neocortical assemblies can be synchronously transferred to the associative networks of the hippocampus (Sirota et al., 2008). Thus, we focused our attention on the gamma rhythm particularly in the VC. High-frequency oscillations in neocortical structures are known to provide temporal windows that bind coherently cooperating neuronal assemblies for the representation, processing, sensory binding and storage of information (Singer, 1993; Buzsáki et al., 1994; Gray, 1994; Traub et al., 1999; Engel et al., 2001). Here we examined the concurrent changes in hippocampal theta- and cortical gamma spectral powers and compared their correlation during the presentation of novel and non-novel stimuli.

The entorhinal cortex provides the major cortical input to the hippocampus, with entorhinal-dentate gyrus interactions serving to filter the entry of sensory information and selectively gate learning-induced modification of synaptic weights in the hippocampal formation (Moser and Moser, 1998; Lisman, 1999; Steffenach et al., 2005). As the dentate gyrus (DG) comprises the first component of the polysynaptic pathway in the hippocampal formation, we investigated the effects of VC activity on the DG. The main hypothesis of the present study is that visually-driven novel exploration can induce synchronous alterations of cortical and hippocampal field activity, which promote DG synaptic plasticity. Our data reveal that synaptic activity in the primary VC of adult, freely behaving rats, generates theta rhythm augmentation and subsequent fluctuation of hippocampal excitability.

\section{MATERIALS AND METHODS}

All experimental and surgical procedures were carried out in accordance with the guidelines of the German Animal Protection Law and were approved by the North Rhine Westphalia State Authority.

\section{SURGICAL PREPARATION}

Under sodium pentobarbitone anesthesia (Nembutal; $40 \mathrm{mg} / \mathrm{kg}$, i.p.), 7- to 9-week-old male Long Evans rats (Charles River Breeding Laboratories, Sulzfeld, Germany) underwent implantation of a monopolar recording and a bipolar stimulating electrode (made from $0.1 \mathrm{~mm}$ diameter Teflon-coated stainless steel wire) in the visual system and also in the ipsilateral hippocampus.

For the recording electrode in the visual system, a drill hole was made, (1-mm in diameter), 7.1-7.3 $\mathrm{mm}$ posterior to bregma and $3.4-3.6 \mathrm{~mm}$ lateral to the midline, corresponding to the primary VC in the rat (Zilles, 1985). A second drill hole ( $3.8 \mathrm{~mm}$ posterior to bregma, 3.3-3.4 mm lateral to midline) was made for the bipolar stimulating electrode in a location that corresponds to dorsal lateral geniculate nucleus (dLGN). The dura was pierced through both holes using a needle. The recording electrode was lowered 200-250 $\mu \mathrm{m}$ from the dural surface to reach the supragranular layer 2/3. A stimulating electrode was positioned in the dLGN ipsilateral to the hemisphere from which $\mathrm{VC}$ recordings were obtained. The depth was $3.7-4.7 \mathrm{~mm}$ from the dural surface, corresponding with the position of the anterior pole where optic radiation fibers are bundled in close proximity (Hughes, 1977; Molnar et al., 1998). Final positions of the stimulating and recording electrodes were then determined by maximizing the amplitude of the field potential (FP) recorded in the $\mathrm{VC}$ in response to electrical stimulation of dLGN. Monopolar recordings from primary VC were made relative to ground and reference screws inserted into the contralateral parietal and frontal bones. For recordings from the hippocampus, a recording electrode was placed in the granule cell layer of the DG and a stimulating electrode was placed in the medial perforant path. A drill hole was made ( $1.0 \mathrm{~mm}$ diameter) for the recording electrode (3.1 mm posterior to bregma, $1.9 \mathrm{~mm}$ lateral to the midline) and a second drill hole ( $1.5 \mathrm{~mm}$ diameter, $6.9 \mathrm{~mm}$ posterior to bregma and $4.1 \mathrm{~mm}$ lateral to the midline) for the stimulating electrode. Electrodes were lowered until a characteristic positivegoing field excitatory postsynaptic potential (fEPSP) upon which a negative going population spike (PS) was superimposed, was identified and optimised.

The thalamic and hippocampal coordinates were chosen for the most distanced configuration in order to prevent a current leak from the dLGN electrode to the perforant path or the DG. The lack of direct current stimulation of the DG via the dLGN electrode was evident with the absence of hippocampal fEPSP after thalamic test-pulse stimualtion. Once verification of the location of the electrodes was complete, the entire assembly was sealed and fixed to the skull with dental acrylic (Paladur; Heraeus Kulzer, GmbH, Wehrheim, Germany). The animals were allowed 10 days to recover from surgery before experiments were conducted. Throughout the experiments the animals could move freely within the recording chamber $(40 \mathrm{~cm} \times 40 \mathrm{~cm} \times 40 \mathrm{~cm})$, as the implanted electrodes were connected by a flexible cable and swivel connector to a stimulation unit and amplifier. Apart from the insertion of the connector cable at the start of the experiment, disturbance of the animals was kept to an absolute minimum. Throughout the experiments the electroencephalogram (EEG) of each animal was continuously monitored.

\section{MEASUREMENT OF EVOKED POTENTIALS}

The fEPSP slope was used as a measure of excitatory synaptic transmission in the VC and DG. To obtain these measurements, an evoked response was generated in the dLGN and medial perforant pathway (MPP), respectively, by stimulating at low frequency $(0.025 \mathrm{~Hz})$ with single biphasic square wave pulses of $0.1 \mathrm{~ms}$ duration per half wave, generated by a constant current isolation unit. For each time-point measured during the experiments, five records of evoked responses were averaged. For hippocampal recordings both fEPSP slope and PS amplitude were monitored. The characteristic field response of the DG granule cells to perforant path stimulation consisted of a positive-going fEPSP with a superimposed negative going PS. The fEPSP slope and PS amplitude were measured for each response. The slope function of the FP was taken as the slope between the first minimum and the first maximum of the potential. This value reflects the granule cell depolarization. The PS amplitude was measured as the absolute value between the first maximum and the following second minimum of the evoked 
potential in $\mathrm{mV}$. This amplitude reflects the number of granule cells firing, as well as the synchrony with which they fire. For VC recordings, the slope and the amplitude of the stimulus-induced negative fEPSP, measured from the peak of the first positive deflection of the evoked potential to the peak of the following negative potential. By means of input-output curve determination, the maximum fEPSP was found, and during experiments all potentials used as baseline criteria were evoked at a stimulus intensity that produced $40 \%$ of this maximum $(100-400 \mu \mathrm{A})$.

The animals were kept in a 12:12 h light-dark cycle of low (0.110 lux) and high (350-400 lux) illuminance conditions. Circadian time was based on Zeitgeber time (ZT), with ZT 0 denoting light on, and ZT 12 denoting light off. Twenty-four hours later, measurements were recorded at the same circadian time and conditions. Theta-burst stimulation (TBS) consisted of 10 bursts at $5 \mathrm{~Hz}$, with each burst containing 5 pulses at $100 \mathrm{~Hz}$, given four times with a 10 -s interval between each train of 10 bursts. In all cases, the stimulus amplitude was the same as that used for recordings.

\section{RECORDING ENVIRONMENT AND NOVEL OBJECTS}

The recording chamber consisted of a grey Perspex box $(40 \mathrm{~cm} \times 40 \mathrm{~cm} \times 40 \mathrm{~cm})$ with a translucent red perspex door. Novel objects consisted of large objects $(3 \mathrm{~cm} \times 10 \mathrm{~cm} \times 10 \mathrm{~cm})$ with different colours (black, white, green) and shapes (cylindric or square). Rearing was considered as placement of animal's front limbs onto the surface of the object. Illuminance of objects and environments was assessed using a Luxmeter (MS-1300) with a sensitivity range of $0-50000$ lux. The luxmeter was placed on the floor of the recording chamber and pointed at the light source. The sensor unit possessed a photosensitive surface, which recorded the total amount of falling light.

\section{DATA ANALYSIS OF ELECTROPHYSIOLOGICAL DATA}

The baseline fEPSP data for VC and DG were obtained by averaging five sweeps at 40-s intervals, every $5 \mathrm{~min}$ over a period of $30 \mathrm{~min}$ after stimulation of the dLGN and of MPP, respectively. The LGN test-pulse-evoked EPSP in VC preceded MPP test-pulse-evoked EPSP in the DG by $10 \mathrm{~s}$ except for the application of TBS where the burst of pulses was delivered only to dLGN. Preliminary tests revealed no influence of the pairing interval $(2,4,6,8$ and $10 \mathrm{~s})$ and sequence on the amplitude of the evoked fEPSP. Electrophysiological data were then expressed as mean percentage of baseline fEPSP reading \pm standard error of the mean (SEM). Statistical significance was estimated by using ANOVA and post hoc Student's $t$ - and LSDtests and by post hoc Student's $t$-tests. The probability level interpreted as statistically significant was $p<0.05$. For the correlation analyses we used Pearson's coefficient of comparison.

\section{DATA ANALYSIS OF NETWORK ACTIVITY}

An intracortical EEG was obtained by means of recordings obtained from the supragranular layer $2 / 3$ of primary $\mathrm{VC}$ and from the granule cell layer in the DG. EEG was sampled at $0.5 \mathrm{kHz}$ and stored on a hard disc for further off-line analysis. In order to evaluate delta $(1-3.5 \mathrm{~Hz})$, theta $(4-10 \mathrm{~Hz})$, alpha $(10-13 \mathrm{~Hz})$, beta1 $(13.5-18 \mathrm{~Hz})$, beta2 $(18.5-30 \mathrm{~Hz})$ and gamma $(30-100 \mathrm{~Hz})$ oscillatory activity during the course of experiment, 4-s long epochs, $1 \mathrm{~s}$ after each test-pulse were selected. Fourier analysis of artefact-free epochs was performed with Hanning window function using "Spike2" software (Cambridge Electronic Design). The absolute values of spectral power for each individual animal were transformed into relative ones (with mean value for baseline preexploration period - the first six time points taken as $100 \%$ ) that were used further for statistics. For each time point, the results of Fourier analysis of five epochs were averaged. Theta activity was measured by the ratio between the relative values of theta and delta spectral powers (T-ratio). The statistical treatment and analysis of data included the calculation of descriptive statistics (mean, SEM) and analysis of variance. For the correlation analyses we used Pearson's coefficient of comparison. Power spectrograms are average of four $10 \mathrm{~s}$-theta periods, which were evaluated and averaged per each animal. The statistical comparison between groups was based on paired student's $t$-test. EEG signal frequency analysis was done using MATLAB's Signal Processing Toolbox (MATLAB, Natick, MA, USA) where the power was calculated by extracting the short-time Fourier transform from a signal (hanning window of $2 \mathrm{~s}$, overlap of $1 \mathrm{~s}$, sampling frequency $100 \mathrm{~Hz}$ ) and interpolated into a continuous power spectrum aligned to the haemodynamic traces. Information was displayed as the magnitude of the time-dependent Fourier transform versus time in a color gradient graph.

\section{DATA ANALYSIS OF CROSS-CORRELOGRAMS}

Electroencephalogram and FP waveforms were analyzed using the Spike2-software package and Matlab. Phase shifts in crosscorrelograms were calculated with respect to the deviation of the first peak from zero (Seidenbecher et al., 2003). Twelve 10 s-theta periods each were evaluated and averaged for each group. The theta epochs for the novelty exploration- and re-exposure groups were taken immediately after the novel objects presentation. The theta epochs for the REM period-, non-exploration theta groups were taken from the highest theta spectral power epoch of each animal and each behavioral state. The REM epochs (non-aroused theta periods) were defined as theta epochs preceded and followed by large irregular activity, which is a characteristic feature of slow-wave sleep (SWS). Theta epochs unrelated to sleep (not linked to SWS) or object exploration were defined as baseline theta (aroused theta periods). Statistical analysis of electrophysiological and behavioral data was done with Student's unpaired T-test, keeping the criterion for significance at $p<0.05$.

Concurrently, to measure the stability of the theta rhythm, the peak-to-trough correlation coefficient was calculated from the cross-correlogram by subtracting the minimum negative value of the correlation coefficient from the maximum of the first positive peak (Madriaga et al., 2004) over the first 3 lags; each lag between 100 and $150 \mathrm{~ms}$ (theta range). Statistical comparisons between experimental conditions were made using a $t$-test or one-way ANOVA if the data were normally distributed and had equal variance.

An additional approach in analyzing the phase coherence in cross-correlograms was the estimation of the correlation coefficient between two signals recorded simultaneously (ventral root recordings), calculated at time $t=0$ (Pearlstein et al., 2005). The mean correlation coefficient value is the average of all correlation coefficients observed in each experiment for a given experimental 
condition. Positive and negative values of the mean correlation coefficient were representative of synchronous and out-of-phase signals, respectively (Pearlstein et al., 2005).

In cross-correlation analysis, waveforms that alternate are out-of-phase from each other and will have a Negative relationship, whereas waveforms that are synchronous will be in-phase and have a Positive relationship. The strength of the relationship between two waveforms will be perfect at \pm 1 and will diminish to a minimum when approaching 0 . A high degree of symmetry or stability along the $x$-axis indicates a stable relationship between the two waveforms. However, as the relationship between waveforms varies, therefore creating decreasing correlation values beyond zero lag, this would indicate less stability in the relationship.

\section{POSTMORTEM VERIFICATION OF ELECTRODE SITE}

At the end of the study, brains were removed for histological verification of electrode localization. Brain sections $(16 \mu \mathrm{m})$ were embedded in paraffin, stained according to the Nissl method using $1 \%$ toluidine blue, and then examined using a light microscope. Brains in which an incorrect electrode localization was found were discarded from the study.

\section{RESULTS}

\section{CORTICAL AND HIPPOCAMPAL OSCILLATIONS ARE CONCURRENTLY INTERLINKED DURING NOVELTY EXPLORATION}

To examine naturally evoked frequency oscillations in the primary VC and DG in adult freely moving rats, animals underwent implantation of monopolar recording electrodes at the surface of the VC and in the granule cell layer of ipsilateral DG (Figure 1A). The first goal of our study was to clarify which neuronal oscillations concurrently change during active exploration in both regions. An intracortical EEG was recorded in parallel from the VC and DG of naive rats following placement in a recording box with low light conditions (0.1-10 lux). After the dark-light diurnal shift the baseline recording continued in high illuminance (350-400 lux) for $180 \mathrm{~min}$ (Figures 1B,D). Subsequently, novel objects were placed in the recording boxes and during their exploration, an increase in cortical gamma (30-100 Hz) spectral power was evident (Figure 1B, black symbols). In order to distinguish the magnitude of cortical high-frequency oscillations associated with the acquisition of new sensory stimuli (Singer, 1993; Gray, 1994; Fries et al., 2001), the same novel objects were presented in the same configuration 5 days later (re-exposure period) under the same recording protocol (Figure 1B, white symbols). The animals' behaviour was characterised by decreased exploratory activity reflected by a lower amount of rearing activity per object ( $t$-test, $n=4, p<0.05$; Figure 1C). Gamma spectral power during the re-exploration also showed significantly diminished values (ANOVA, $F=3.27, p<0.01, n=4$ ), compared to the novelty exposure experiment (Figure 1B). Oscillatory activity in the ipsilateral hippocampus shared similar patterns during the novel exploration and the re-exposure. Concordantly, we observed increased values of theta $(4-10 \mathrm{~Hz})$ and gamma hippocampal rhythms $(30-100 \mathrm{~Hz})$. We found an increase, during the novelty exposure to the novel objects, in DG theta-ratio (Figure 1D, black symbols), in parallel with an augmentation of VC gamma oscillations. Theta-ratio represents the ratio between the relative values of theta and delta spectral powers (Harris et al., 2002). The re-exposure to the same objects resulted in no increase of theta-ratio compared to the preceding novelty exposure (ANOVA, $F=2.59, p<0.05, n=4$, white symbols). As the link between locomotion and hippocampal oscillations is well established (Bland, 1986; Sainsbury et al., 1987) the focus of our experiments was to investigate the link between hippocampal and cortical rhythms. In addition to the theta and gamma bands, the other measured frequencies: alpha $(10-13 \mathrm{~Hz})$, beta $1(13.5-18 \mathrm{~Hz})$ and beta $2(18.5-30 \mathrm{~Hz})$ also revealed a tendency towards a higher spectral power during the novel exposure compared to the re-exposure, but only cortical alpha and betal underwent a significant increase (ANOVA, $F=3.24, p<0.05$, for alpha and $F=2.03, p<0.05, n=4$, for beta1, data not shown).

Our EEG analyses demonstrated a significant correlation between gamma spectral power in both VC and DG for the novelty exposure (Pearson, $n=4, p<0.05, r=0.108$, data not shown) as well as for the re-exposure (Pearson, $n=4, p<0.05, r=0.109$, data not shown). Most importantly, the correlation between VC gamma and DG theta-ratio was significant only during the presentation of novel objects (Pearson, $n=4, p<0.001, r=0.2266$; Figure 2A) but not during the subsequent re-exposure (Pearson, $n=4, p>0.05$, $r=0.0023$; Figure 2B). This experiment proposes a direct functional relationship between high-frequency oscillatory activity in primary VC, and DG theta rhythm.

To demonstrate that the oscillatory changes of VC and hippocampus are predominantly interlinked during the processing of novel visual stimuli, we compared the synchronization in both regions during the novelty exploration and later during object re-exposure (Figure 2). The EEG recordings in VC (Figure 1E) and hippocampus (Figure 1F) suggest a negative relationship between the peaks and troughs of the slow spectral ranges in both regions (Figure 3A). Cross-correlation analyses confirmed a highly negative relationship between hippocampal and cortical oscillations (Figure 3B). Re-exposure to the objects resulted in a non-significant increase of the mean correlation coefficient calculated at time $t=0$ and concurrently in a significant decrease of peak-to-trough correlation coefficient ( $t$-test, $n=4, p<0.05$; Figure 3B). Therefore, during the re-exposure, the stability of the synchronization in theta range is diminished and this observation is confirmed by the drop of the absolute theta spectral powers in VC ( $t$-test, $n=4, p<0.05$; Figure 3C) and DG ( $t$-test, $n=4$, $p<0.01$; Figure 3D). The reduced theta/delta ratio in DG reveals a decline in exploration-related hippocampal oscillatory activity ( $t$-test, $n=4, p<0.05$; Figure $3 \mathrm{E}$ ). This decline can be visualized in a power spectrogram when compared to the novel exploration period (Figures $4 \mathrm{~A}, \mathrm{C}$ ). The changes in theta range are less apparent for VC (Figures 4B,D). As a control measurement of the theta parameters in both regions, we analyzed EEG epochs during non-novelty-related theta periods in aroused- (baseline theta; Figure 5) and non-aroused state (REM sleep; Figure 6). The waveform synchronization during aroused behavioral state of freely behaving animals habituated with the recording box (Figure 5A) has the same cross-correlation values as the novelty explorationinduced oscillations (Figure 5B). The difference in both groups is demonstrated by significant changes in the amplitude of the low-spectrum oscillations recorded in VC ( $t$-test, $n=4, p<0.001$; Figure 5C) and DG ( $t$-test, $n=4, p<0.001$; Figure 5D). In addition a theta shift towards a higher frequency $-9 \mathrm{~Hz}$ is evident in 

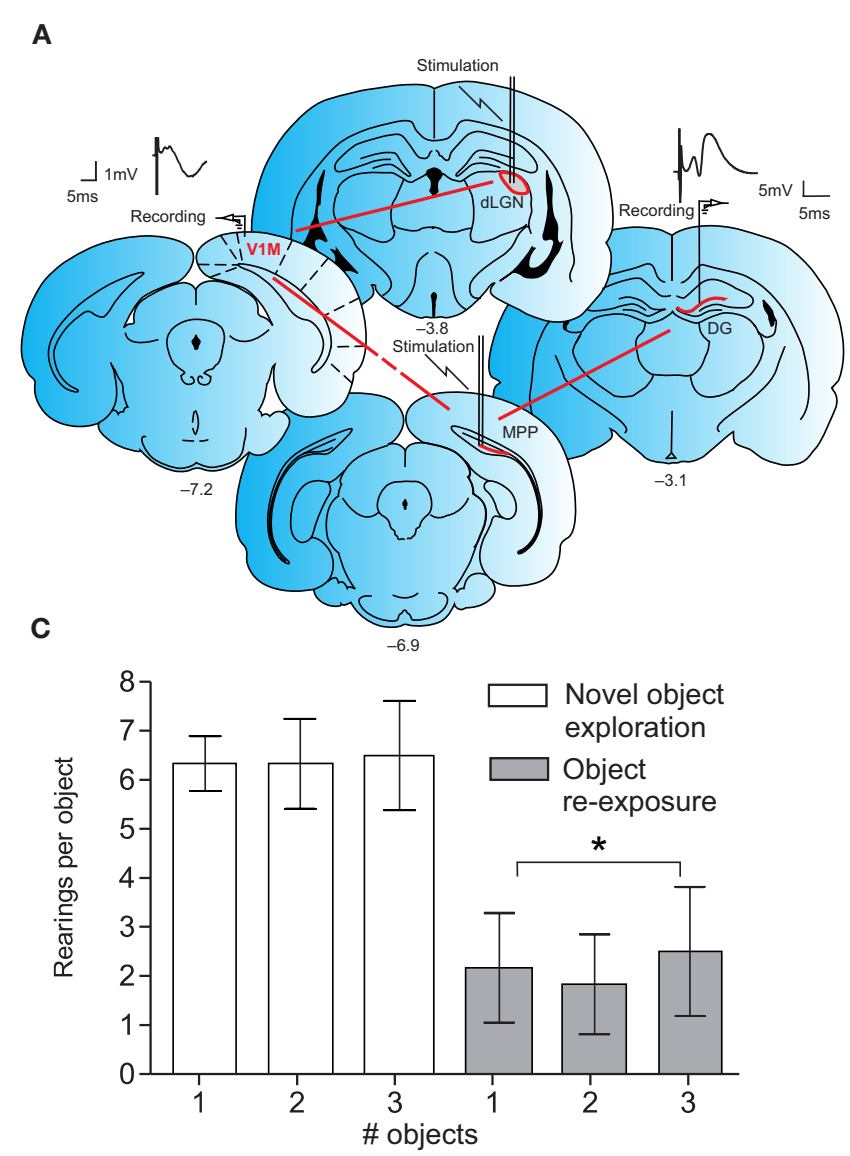

$\mathbf{E}$

Visual cortex novelty

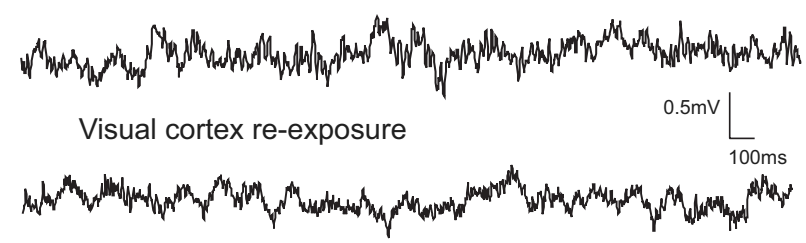

FIGURE 1 | Novelty-evoked high-frequency cortical oscillations parallel the induction of theta rhythms in the dentate gyrus. (A) Schematic presentation of cortico-hippocampal information flow and illustration of the location of implanted electrodes. The red arrows indicate the direction of synaptic transmission and the interrupted red arrow implies polysynaptic transmission between the indicated structures. The bipolar stimulating electrode in $\mathrm{dLGN}$ and the monopolar recording electrode in the primary visual cortex [V1M] represent the thalamocortical contact. Stimulation and recording electrodes in the MPP and the dentate gyrus (DG), respectively, show the entorhino-hippocampal interaction. Analog traces represent the evoked fEPSPs in the visual cortex (VC) and DG. Electrodes' anterior-posterior coordinates relative to bregma are indicated below the drawings. (B) Novelty exploration (the presentation of the objects is indicated by the gray rectangle) leads to increased gamma oscillations in VC $(n=4)$. During re-exposure to the objects a reduction in visual cortex gamma activity was observed $(n=4)$. To exclude the interference of diurnal fluctuation of basal synaptic transmission in VC on the novelty protocol, baseline
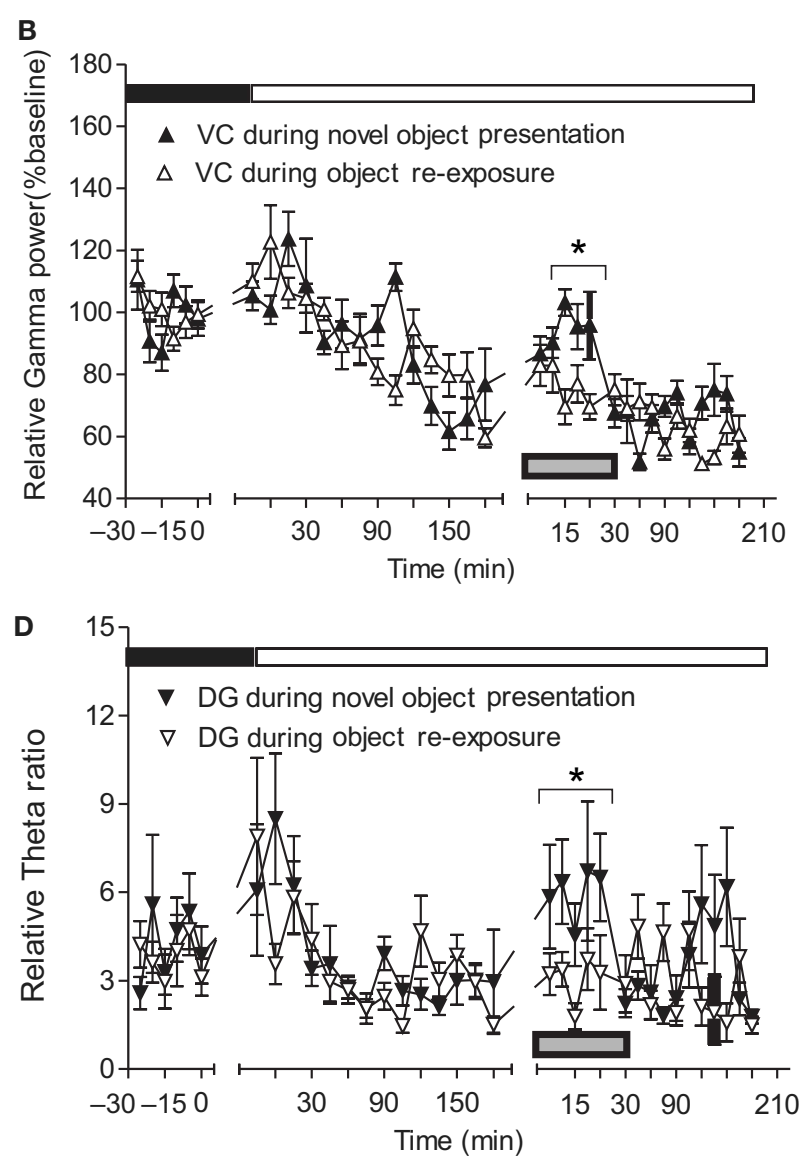

F

Dentate gyrus novelty

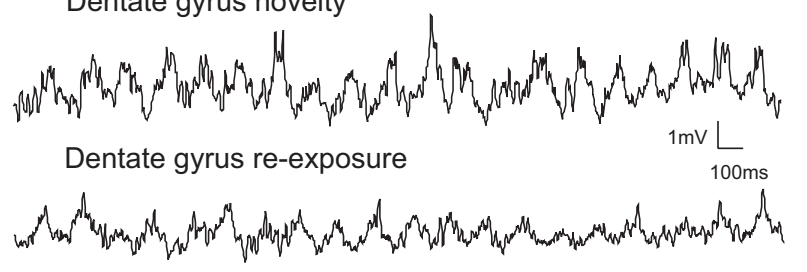

recordings were taken during the circadian illuminance shift. Higher levels of gamma frequencies were observed in VC during the dark phase (indicated with black rectangle) and in the beginning of the light phase (white rectangle) of the night:dark cycle; standard errors of the mean values were used for the error bars; ${ }^{*} p<0.05$. (C) Behavioral arousal in response to novel object exploration (white) and re-exposure to the same objects 5 days later (grey) measured by number of rearings per object. (D) Comparison of theta-ratio in dentate gyrus during exploration of novel objects $(n=4)$ and the re-exposure $(n=4)$ to the objects in the same configuration. The decrease of cortical gamma activity is paralleled by a drop in hippocampal theta-ratio. A transient increase in theta activity in the dentate gyrus also occurred as a consequence of the illuminance change; * $p<0.05$. (E) Original traces of field potential recordings in the VC during the novelty exploration-induced theta period (upper trace) and reexposure (lower trace). (F) Original traces of field potential recordings in the DG during the novelty exploration-induced theta period (upper trace) and reexposure (lower trace). the baseline theta group compared to the $8 \mathrm{~Hz}$ seen in the novelty theta group. Importantly the DG theta-ratio in both groups does not differ ( $t$-test, $n=4, p<0.001$; Figure 5E), showing that both behavioural states share proportional changes in delta and theta frequencies. In order to clarify the specificity of cortical and hippocampal oscillatory parameters during novelty exploration 


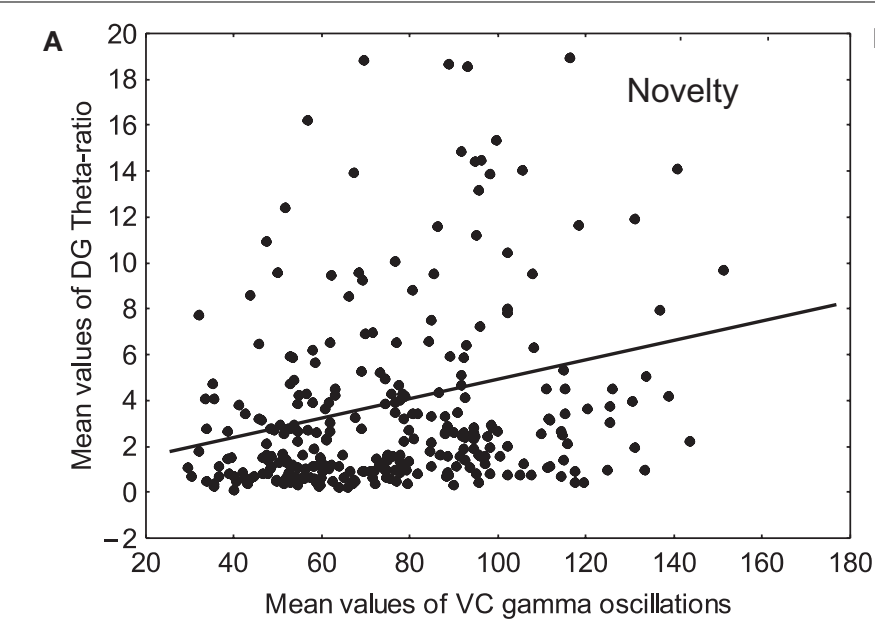

FIGURE 2 | Gamma oscillations in the visual cortex and theta-ratio in the dentate gyrus correlate during novelty exploration. (A) Significant (Pearson, $n=4, p<0.0001, r=0.2266$ ) values between visual cortex (VC) gamma oscillations

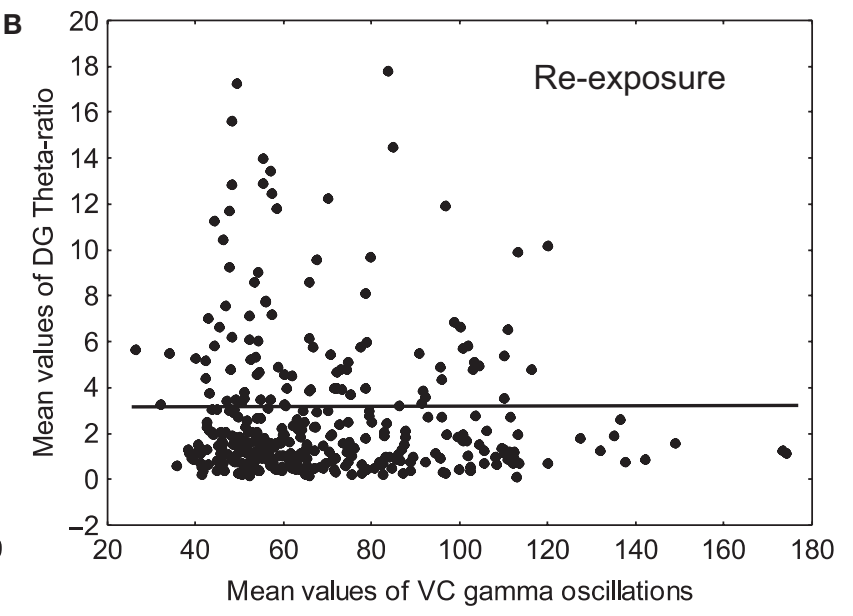

and dentate gyrus (DG) theta-ratio were observed only during the introduction of the novel objects. (B) 5 days later during the re-exposure, the correlation values are below the level of significance (Pearson, $n=4, p>0.05, r=0.0023$ ).
A

A DG

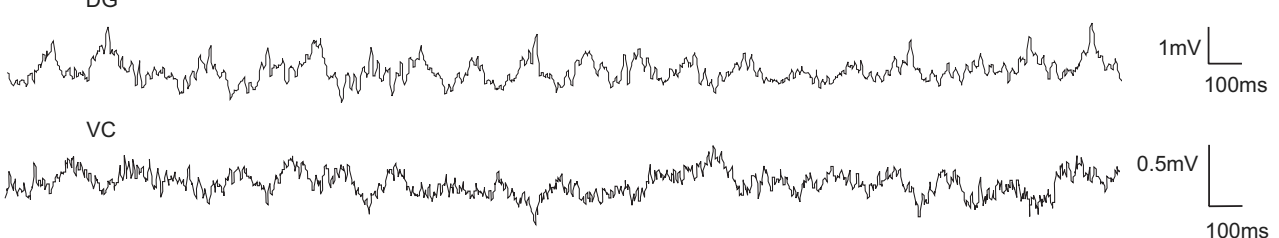

B

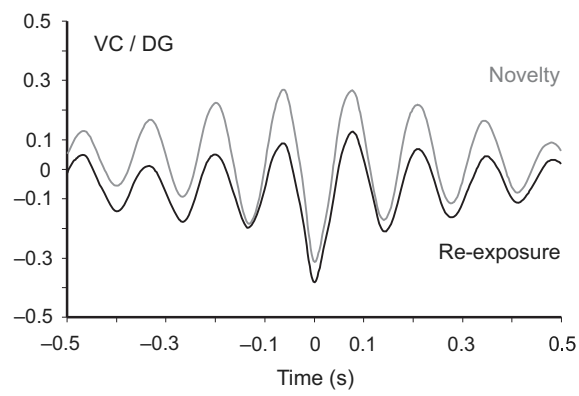

C

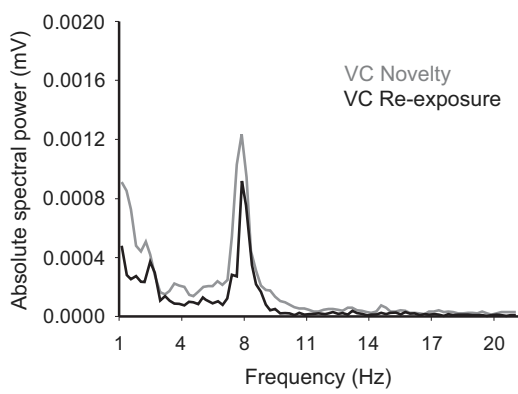

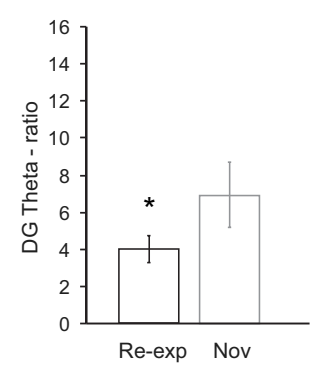

D

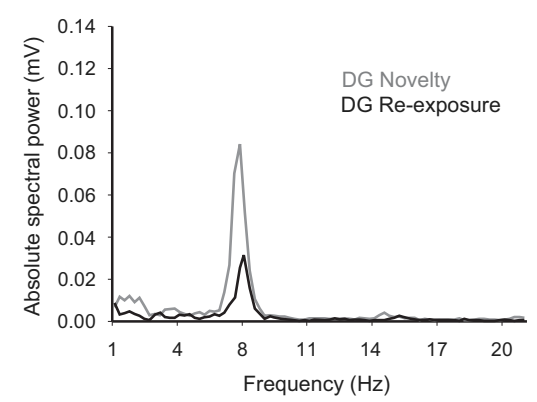

FIGURE 3 | Neural activity in the DG and VC during novel exploration and re-exposure theta periods. (A) Original traces of field potential recordings in the DG (upper trace) and the VC (bottom trace) during re-exposure theta period. (B) Cross-correlation analyses of activities in the DG and the VC during the repeated presentation (black trace, $n=4$ ) reveal a lower level of synchronization in comparison to the novel presentation (grey trace, $n=4$ ), estimated by peak-to-trough correlation coefficient. (C) Examples of visual cortex and (D) dentate gyrus spectral powers during the re-exposure (black trace) and the novelty (grey trace) periods. Lower values are detected for the re-exposure in the range of $8 \mathrm{~Hz}$ frequency. (E) Theta-ratio (relative theta-delta ratio) in the reexposure period (black bars) is lower in comparison to the novelty (grey bars) values; * $p<0.05$. we also analyzed the theta periods of non-aroused REM state (Figure 6A). Interestingly the profile of the REM theta period is characterized with opposite changes in comparison to baseline theta group. The altered synchronization is demonstrated with a phase shift at time 0 (Figure 6B). Although the peak-to-trough correlation coefficient is higher in REM theta periods ( $t$-test, $n=4, p<0.05$; Figure 6B) the phase coherence is shifted and the synchronization between VC and DG is not locked in time. The mean correlation coefficient calculated at time $t=0$ is higher for novelty group ( $t$-test, $n=4, p<0.05$; Figure 6B). At the same time theta spectral value in VC is the same as for the novelty theta periods (Figure 6C) and in DG is even higher ( $t$-test, $n=4, p<0.05$; Figure 6D), paralleled by increased theta-ratio ( $t$-test, $n=4$, $p<0.05$; Figure 6E).Altogether these data reveal that noveltyinduced theta periods underlie a unique combination of waveform synchronization, oscillatory spectral powers and theta-ratio, that 


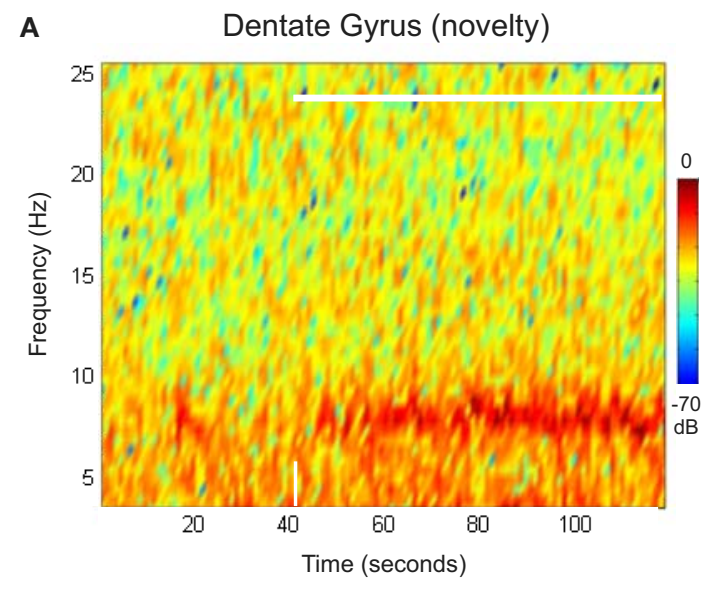

。

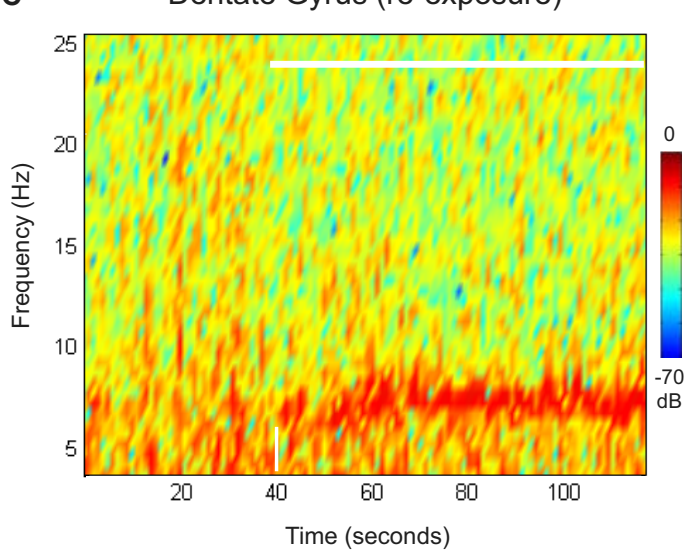

FIGURE 4 | Spectral powers of DG and VC during novel exploration and reexposure theta periods. Representative colour-coded power spectrograms demonstrate dentate gyrus (A) and visual cortex (B) theta activity in a frequency band of 4-10 Hz during the novel objects presentation (white horizontal bar) and pre-exploration phase. Note the theta power peaks featuring in the range about

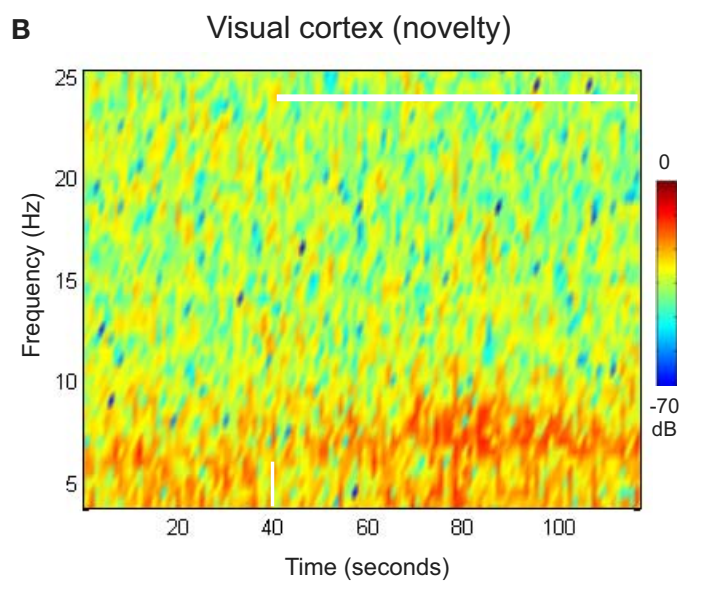

D Visual cortex (re-exposure)

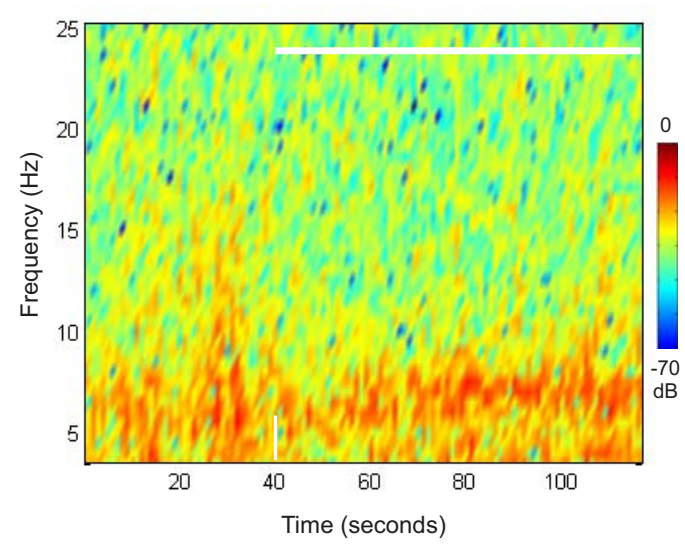

$8 \mathrm{~Hz}$ (in brown colour, at around 70-80 s) for the dentate gyrus after the objects presentation. These peaks are absent during the re-exposure period (C). Less visible changes in the theta range are available for the visual cortex re-exposure period (D). The time-point of object-presentation is marked with a white vertical arrow. are not found in either in the baseline-theta, or REM theta periods that occur during re-exposure. Furthermore, only the novelty theta group is characterized by simultaneously high values of EEG synchronization in VC and DG, high theta spectral powers in both regions, and high theta-ratio.

\section{HIGH-FREQUENCY STIMULATION OF THE LATERAL GENICULATE NUCLEUS EVOKES A LONG-TERM ENHANCEMENT OF GRANULE CELL EXCITABILITY IN THE HIPPOCAMPUS}

The next target of our investigation was the hippocampal synaptic response to novel stimuli and the ability to amplify the effect of neocortical input on hippocampal plasticity through a thalamocortical stimulation protocol. First, we examined the hippocampal fEPSP in relation to novelty exploration as a baseline comparison for the stimulation-induced plasticity alterations. Many conflicting reports exist that describe that exploration increases (Stanton and Sarvey, 1987; Kithigina et al., 1997; Davis et al., 2004) or decreases (Sharp et al., 1989; Green et al., 1990) the PS amplitude, and which discuss whether exploration also affects the fEPSP. These differences are probably related to variations in the behavioral protocols used, the time-scale of recordings and the fEPSP measurement parameters. Therefore, we first examined the changes in the DG field response to entorhinal stimulation during novelty exploration under our recording conditions. Investigations of the granule cell field response (Figure 7A) revealed a novelty-related fluctuation of the fEPSP. The introduction of novel objects was characterized by a shortterm depression of the PS amplitude in the DG ( $t$-test, $p<0.05$, $n=6$ ), that lasted for $20 \mathrm{~min}$ and was followed by a rebound tendency towards short-lasting potentiation. The PS fluctuation in our experiments occurred in the absence of a comparable change in the fEPSP slope (Figure 7C), suggesting an occurrence of EPSP-spike (E-S) depression/potentiation. The depression period was paralleled by a transient increase of the DG T-ratio (ANOVA, $F=3.51, p<0.01, n=6$; Figure 7A).

In order to amplify the impact neocortical high-frequency oscillations on hippocampal plasticity we activated a larger portion of VC neurons (than that elicited by visual exploration) through use of TBS of the dLGN. TBS of the dLGN induces LTP in the rat VC under anaesthesia (Heynen and Bear, 2001) and in awake animals (Tsanov 


$$
\text { A DG }
$$

E

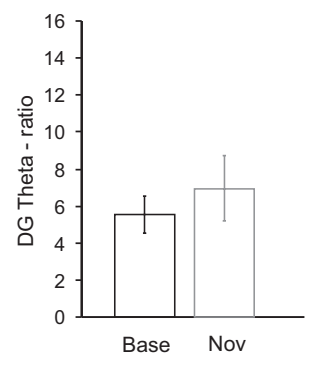

B

C

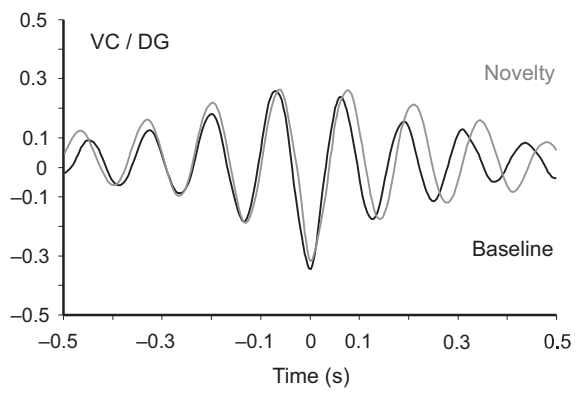

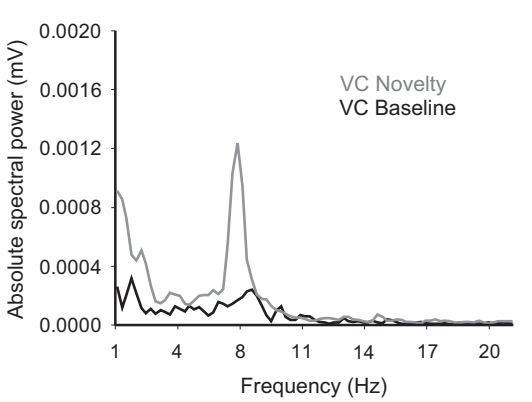

D

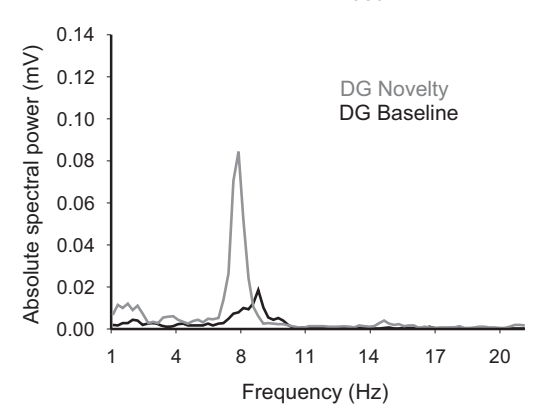

FIGURE 5 | Neural activity in the DG and VC during baseline theta period. (A) Original traces of field potential recordings in the DG (upper trace) and the VC (bottom trace) during baseline theta period (aroused theta, see Materials and Methods). (B) Cross-correlation analyses of activities in the DG and the VC during the baseline theta periods (black trace, $n=4$ ) reveal no significant difference of the waveforms synchronization in comparison to the novelty exploration (grey trace, $n=4$ ). Note a tendency towards a right-shift in the cross-correlogram during novelty exposure that corresponds to slower theta oscillations. (C) Visual cortex and (D) dentate gyrus spectral power examples during the baseline (black trace) and the novelty (grey trace) periods. Higher values are detected for the novelty theta periods in the range of $8 \mathrm{~Hz}$ frequency. Note the difference of the theta peak, which is in the range of 9-10 Hz for the baseline theta periods. (E) Theta-ratio baseline values (black bars) are similar in comparison to the novelty (grey bars) ones.

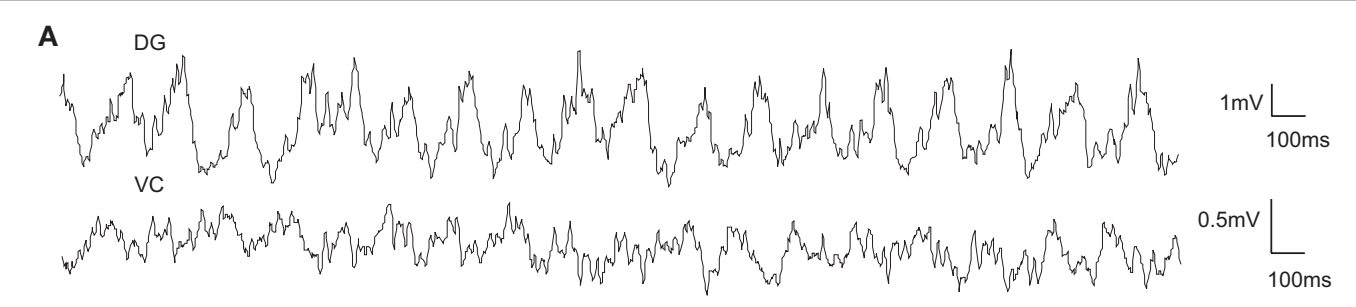

B

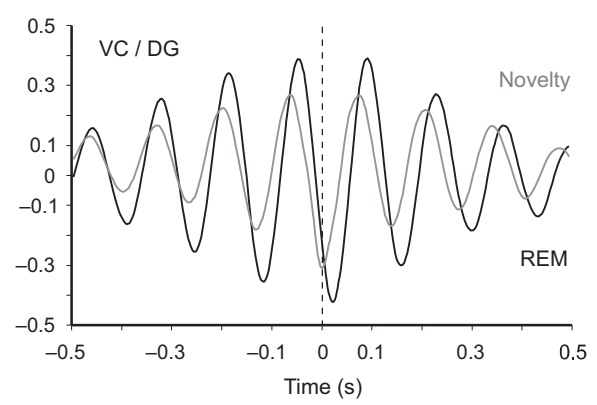

C

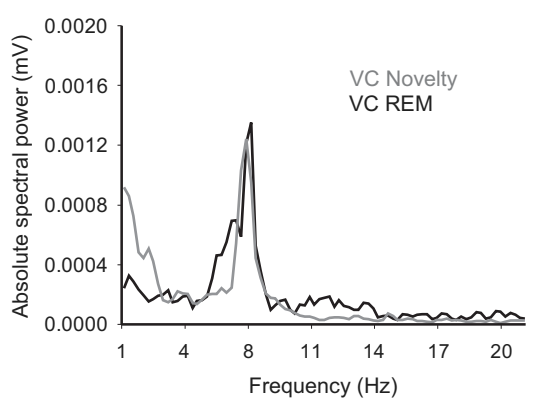

E

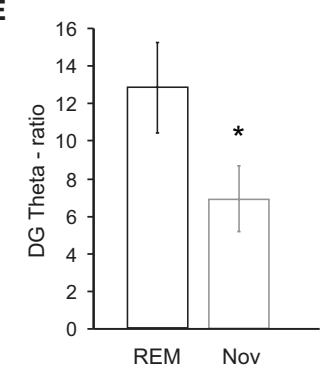

D

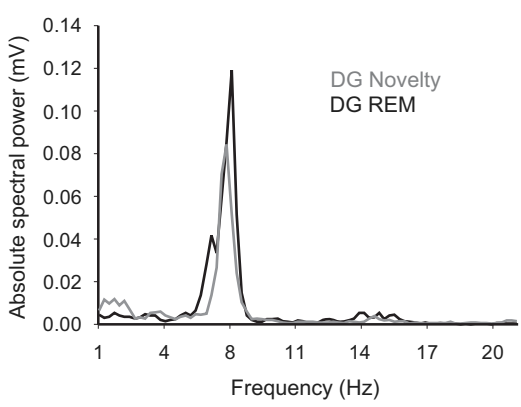

FIGURE 6 | Neural activity in the DG and VC during REM theta period. (A) Original traces of field potential recordings in the DG (upper trace) and the VC (bottom trace) during REM theta period (non-aroused theta, see Materials and Methods). (B) Cross-correlation analyses of activities in the DG and the VC during the REM theta periods (black trace, $n=4$ ) reveal higher peak-to-trough correlation coefficient in comparison to the novelty group (grey trace, $n=4$ ), but lower mean correlation coefficient calculated at time $t=0$ (marked with dotted line). (C) Visual cortex and (D) dentate gyrus spectral power examples during the REM (black trace) and the novelty (grey trace) periods. Higher values for $D G$, but not for $V C$ are detected during the REM theta periods. Note the appearance of additional small theta peak in the range of $7 \mathrm{~Hz}$ for the REM theta periods. (E) Theta-ratio in the REM period (black bars) is higher in comparison to the novelty (grey bars) values; ${ }^{*} p<0.05$. 

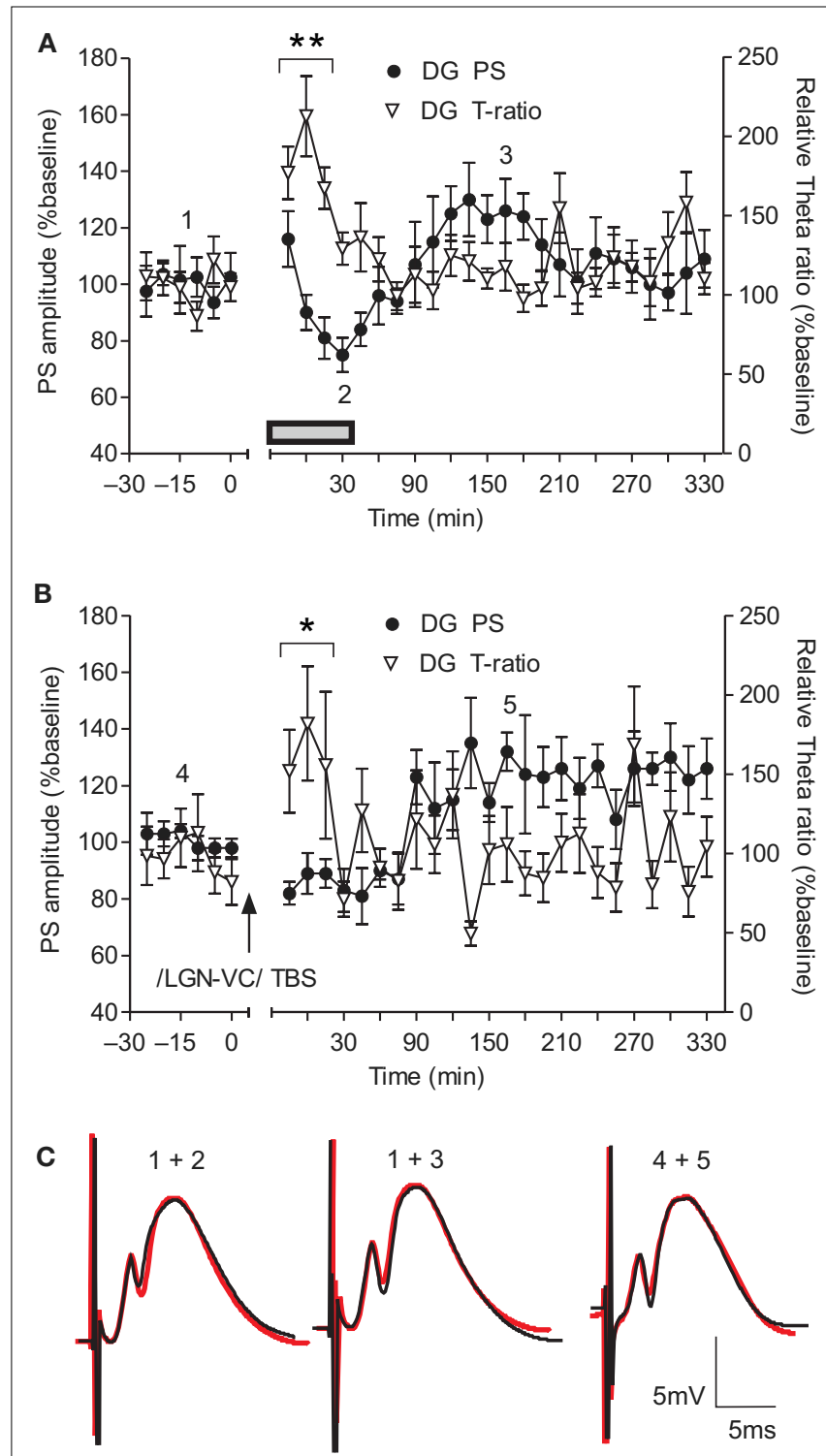

FIGURE 7 | Dentate gyrus excitability changes are paralleled by an increase in theta activity following either novelty exploration or thalamocortical stimulation. (A) Short-term depression occurs in the course of novelty exploration (signified by the grey rectangle), and is followed by a subtle potentiation of the population spike (PS) in the dentate gyrus (DG) $(n=6)$. The immediate response of theta oscillations, expressed by theta-ratio (T-ratio), occurs in parallel with the suppression of dentate gyrus excitability. (B) Theta-burst stimulation of lateral geniculate nucleus (LGN) results in a lateonset enhancement of the population spike response 90 min after the highfrequency protocol $(n=5)$. This potentiation continued until the end of the recording period. Immediately after LGN stimulation an increase in the thetaratio was observed in the dentate gyrus granule cell layer. (C) Analog traces represent an average of 5 field potentials evoked at the points marked in the figures. The red traces reveal the fEPSP profile of recorded potentials during the baseline period (marked with 1 and 4, respectively). Note the increase of PS paralleled by no change of the fEPSP slope. Horizontal bar: $5 \mathrm{~ms}$, vertical bar: $5 \mathrm{mV} ;{ }^{*} p<0.05$ versus baseline 1 .

and Manahan-Vaughan, 2007a). We thus investigated whether the induction of synaptic plasticity in the adult primary VC has any long-term effects on DG synaptic responsiveness.
Medial perforant pathway-evoked field responses were induced in the DG from freely behaving rats in parallel with ipsilateral thalamocortical recordings (Figure 1A). Baseline measurements, for $30 \mathrm{~min}$, in both the VC and DG were followed by stimulation of the dLGN (Figure 7B). We found that TBS given to the dLGN results in a longlasting augmentation of DG population spike amplitude (ANOVA, $F=2.11, p<0.05, n=5$; Figure 7B), preceded by a short-term period with a tendency towards depression. The fEPSP slope was unaffected, suggesting that E-S potentiation occurred (Figure 7C). The onset of the E-S potentiation appeared with about 90 min of delay after TBS to the dLGN. As the theta rhythm was already associated with a suppression of dentate excitability (Figure 7A), we analysed the T-ratio changes throughout the experiment. Notably, we found a significant increase of theta oscillations in the DG induced by TBS stimulation of visual pathway (ANOVA, $F=3.12, p<0.05, n=5$; Figure $7 \mathbf{B}$ ). Furthermore, the theta augmentation temporally coincided with the delay in onset of E-S potentiation, confirming the role of theta oscillations in the regulation of DG excitability.

Theta-burst stimulation to the dLGN also evokes slow-onset potentiation of the fEPSP amplitude in the VC (Tsanov and Manahan-Vaughan, 2007a). We compared the time-course of the potentiation in both the VC and DG during the parallel recordings. The augmentation of the cortical fEPSP response preceded the DG E-S potentiation (Figure 8A; $n=5$ ). Furthermore, a similar magnitude of long-lasting plasticity occurred in both structures. In order to confirm that these parallel potentiations were not independently occurring events, we compared each fEPSP response in the VC with the subsequent DG population spike amplitude (Figure $\mathbf{8 B}$ ) in every recording from all animals. The VC fEPSP and the subsequent hippocampal response correlated positively (Figure 8C; Pearson, $n=5, p<0.0001, r=0.3880)$, indicating that the degree of cortical plasticity was reflected in the degree of changed DG excitability. The consequence of this parallel plasticity is prolonged DG excitability, and hence increased effectiveness of spatial information processing and encoding. Importantly, significant correlation was also evident between VC gamma oscillations and DG theta-ratio (Figure 8D). (Pearson, $n=4, p<0.0001, r=0.2812$ ) Note the similarity of the TBS correlation profile (Figure 8D) and the novelty correlation (Figure 2A), but not the re-exposure correlation (Figure 2B). This finding suggests a link between the sensory-evoked and electricallyinduced oscillatory activity in VC and hippocampus.

\section{DISCUSSION}

Despite significant progress in understanding the cellular basis of cortical and hippocampal synaptic plasticity, the precise interaction between experience-dependent synaptic changes in these brain regions remains unknown. In our study, parallel field recordings from the VC and hippocampus of freely behaving adult rats revealed that the interaction between cortical gamma oscillations and dentate theta rhythm is highly expressed during active visual exploration of novel objects. Theta-burst stimulation of the primary VC drove a long-term enhancement of granule cell excitability in the hippocampus that was proportional to the concurrent potentiation of the cortical response.

The entorhinal cortex provides the major cortical input to the hippocampus, and both structures have been implicated in memory processes (Moser and Moser, 1998). Entorhinal-dentate gyrus 


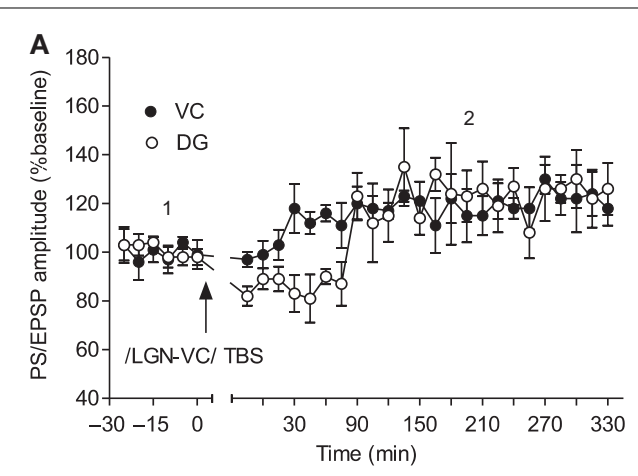

B
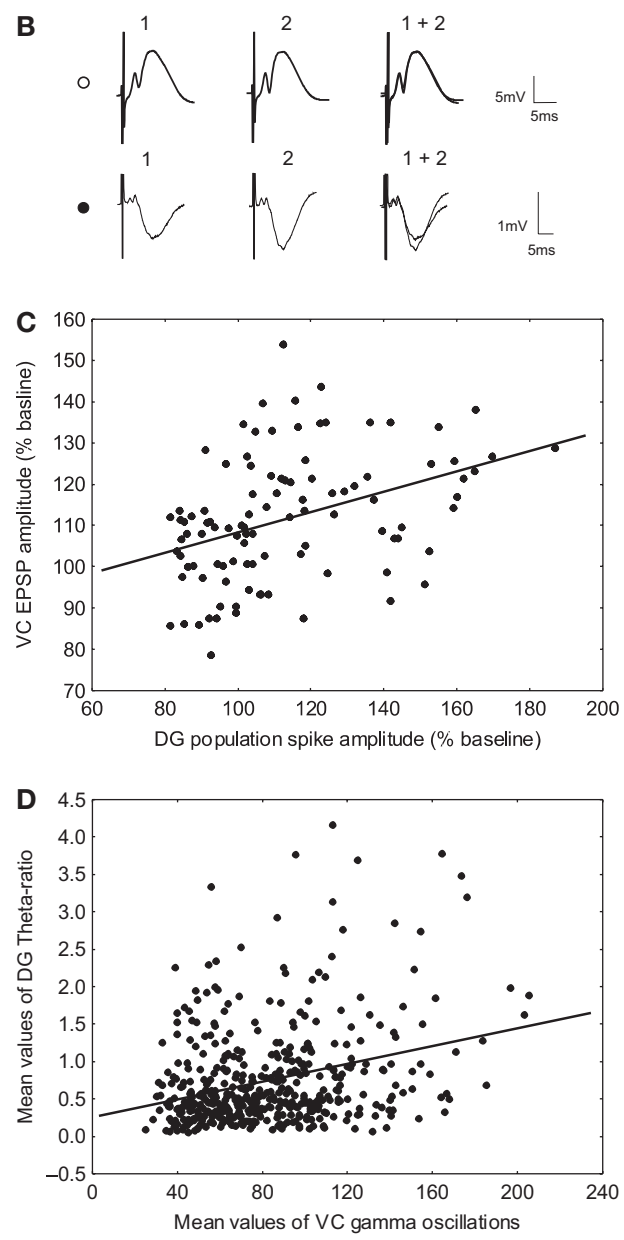

FIGURE 8 | LGN-TBS drives enhancement of the visual cortex EPSP in close correlation with the late-onset dentate gyrus E-S potentiation. (A) Comparison between visual cortex (VC) and dentate gyrus (DG) potentiation onset in response to theta-burst stimulation of LGN $(n=5)$. The late-onset potentiation of the dentate gyrus population spike (PS) was preceded by an augmentation, of the same magnitude (120\%), in the visual cortex field response. The application of TBS to VC induced an initial short-term depression of the DG response. (B) Analog traces represent an average of 5 field potentials evoked at the points marked in the upper figure. (C) A positive correlation between TBS-induced cortical slope enhancement and dentate E-S potentiation, was demonstrated by the comparison of the individual values from all subsequent fEPSP recordings presented in (A). (D) Analysis of the spectral powers from the TBS experiment reveals a significant correlation (Pearson, $n=4, p<0.0001, r=0.2812$ ) between VC gamma oscillations and DG theta-ratio. interactions are believed to filter the entry of sensory information and selectively gate learning-induced modification of synaptic weights in the hippocampal formation (Lisman, 1999). As the DG is the first post of the trisynaptic pathway within the hippocampal formation, our attention was focused on entorhinal-dentate gyrus field alterations in relation to VC activity. Cortico-hippocampal dialogue has been revealed during sleep with strong temporal correlations between primary VC spindles and hippocampal ripples (Ji and Wilson, 2007). Our data demonstrate for the first time that visually-mediated learning triggers EEG synchronization in VC and DG, with a negative phase-shift correlation. In addition, increased theta spectral powers in both regions and high hippocampal thetaratio determined the specific characteristics of novelty-induced theta periods. Such combinations of waveform synchronization, oscillatory spectral powers and theta-ratio can not be evoked in other theta periods. As for the baseline periods, the oscillations of theta range have lower amplitude and a different frequency peak when compared to the novelty-induced theta periods. However, the REM periods are characterized by a high theta spectral power, but with a shifted out-of-phase synchronization between VC and DG. The re-exposure periods differ from the novelty periods with a significantly lower degree of synchronization between VC and DG, lower theta amplitude in both regions and lower DG theta-ratio.

In the sensory cortices, behavioural arousal is characterised by a prominent enhancement of high-frequency oscillations in the gamma frequency range $(40-100 \mathrm{~Hz})$. High-frequency oscillations in cortical structures are believed to provide temporal windows that bind coherently cooperating neuronal assemblies for the representation, processing, storage, and retrieval of information (Buzsáki et al., 1994; Traub et al., 1999; Engel et al., 2001). Of the large family of brain oscillatory patterns, gamma oscillations have received special attention because of their postulated role in sensory binding (Gray, 1994; Singer and Gray, 1995), memory (Fell et al., 2001), attentional selection (Fries et al., 2001), and “conscious" experience (Varela et al., 2001). The gamma cycle is proposed to serve as a fundamental computational mechanism that enables fast processing and flexible routing of activity, supporting fast selection and binding of distributed responses (Fries et al., 2007). A tight relationship exists between changes in gamma oscillatory activity and the direction of change in synaptic strength elicited in the VC (Tsanov and Manahan-Vaughan, 2007b). High-frequency oscillations underlie the processing along the visual pathway (Martinez-Conde et al., 2002) and at the same time are related to synaptic plasticity (Singer, 1993; Steriade, 1999; Wespatat et al., 2004). As the hippocampus processes episodic memory, and receives a large input from the $\mathrm{VC}$ for this purpose, a main focus of this study included the influence of cortical high-frequency oscillations on the hippocampal field alterations. Our data support the hypothesis that gamma activity in the VC correlates with high-and low-frequency oscillations in the DG. Most importantly however, a significant correlation between VC gamma activity and DG theta activity occurs only in the time-course of novel object exploration. In line with our results gamma oscillations in somatosensory cortex were recently shown to be phase locked to hippocampal theta oscillations (Sirota et al., 2008), thus suggesting that theta oscillation entrainment provides a mechanism by which activity in neocortical and hippocampal networks can be temporally coordinated. As a corollary of this, 
high-frequency stimulation of the dLGN that elicits LTP in the VC (Tsanov and Manahan-Vaughan, 2007a) generated a potentiation of gamma activity and an equivalent potentiation of DG theta activity. Furthermore, TBS to the dLGN resulted in a concurrent increase of cortical gamma and dentate theta in a similar manner to the novelty-evoked interregional oscillatory correlation. We propose that high-frequency oscillations in VC lead to alterations of excitability in the hippocampus, which in turn can facilitate the formation of visually-related synaptic memories in the hippocampus. This suggests that VC plasticity lowers the threshold for induction of hippocampal synaptic plasticity and may serve to prime the hippocampus for processing of visual sensory information in a spatial context. Our finding is particularly pertinent in light of reports that novel spatial object exploration elicits synaptic plasticity in the DG and other hippocampal subregions (Kemp and Manahan-Vaughan, 2007, 2008). Non-visual forms of sensory stimuli are also perceived during novel exploration and their impact on hippocampal plasticity (Mouly and Gervais, 2002; Truchet et al., 2002) confirms the model of cortico-hippocampal dialogue in episodic memory formation. Associative olfactory tasks are known to involve a large subpopulation of synapses in the DG during the early stages of the learning process. The early increase of the monosynaptic response in the DG is observed immediately after the first learning whereas the polysynaptic potential increases substantially when rats start to discriminate the learning cues (Mouly and Gervais, 2002; Truchet et al., 2002).

Synaptic plasticity constitutes a cellular mechanism which is thought to underlie information storage in the brain and represents a use-dependent long-lasting increase or decrease in synaptic strength (Barnes, 1995; Bear, 2003). Sensory experience modifies synapses, not only in the hippocampus, but also in the sensory cortex, and particularly in the primary VC (Bear, 2003; Tsanov and ManahanVaughan, 2007a,b). The adult VC is not simply a passive receiver of sensory inputs, but can dynamically amplify the processing of thalamic signals (Sur et al., 2002; Miyamoto and Hensch, 2003). Furthermore, plastic and oscillatory alterations are not simply a passive response of the VC to the light conditions, but crucially depend on visual activity of the animal: the levels of exploratory activity of animals to novel objects under low illuminance (dark phase) is the same for high-contrast and low contrast objects, but the gamma oscillations are significantly higher for the group exposed to the high-contrast object (Tsanov and Manahan-Vaughan, 2007b). Therefore, non-visually guided exploratory activity is insufficient to produce the same amount of augmentation in the VC field response. A central issue of our study was to establish whether the experience-dependent shift in neuronal responses in the visual cortical network is able to influence subsequent (e.g. downstream) stages

\section{REFERENCES}

Abbott,L.F., Varela,J.A.,Sen, K., and Nelson, S.B. (1997).Synaptic depression and cortical gain control. Science 275, 220-224.

Andersen, P., and Moser, E. I. (1995). Brain temperature and hippocampal function. Hippocampus 5, 491-498.

Barnes, C. A. (1995). Involvement of LTP in memory: are we 'searching under the street light'? Neuron 15, 751-754.

of information processing. We demonstrate that long-term synaptic plasticity in VC correlates with an increased dentate PS and this increase is preceded by hippocampal theta augmentation. Our data are in agreement with recent findings that confirm the proposed link between theta rhythm and hippocampal plasticity (Bikbaev and Manahan-Vaughan, 2008; Tsanov and Manahan-Vaughan, 2009). We acknowledge that brain temperature could interfere with shortterm fEPSP fluctuation in the DG during active exploration (Moser et al., 1994; Andersen and Moser, 1995), however, the arena in which exploration took place was (for reasons of its size) not conducive to large physical exertions that could change brain temperature. Theta-burst stimulation to the dLGN resulted in LTP of the visual cortical fEPSP, which was followed by a long-term, slow-onset E-S potentiation of entorhino-dentate transmission. Furthermore, the increased DG population response was closely correlated with the preceding cortical fEPSP augmentation. We observed a striking similarity between hippocampal field changes during novelty exploration and those after TBS of dLGN. Both the post-stimulation period and the novelty exploration were followed by a tendency towards depression of the fEPSP combined with a rebound increase of the theta spectral power. The subsequent late-onset potentiation period was longer and more stable in the group of rats that underwent the stimulation protocol. Taken together, these data suggest that synaptic plasticity and network activity in the primary VC that occur as a consequence of active sensory processing, strongly influence DG excitability and may comprise one of the endogenous triggers for synaptic plasticity in the hippocampus.

\section{OVERVIEW}

Our data present evidence that experience-dependent plasticity of functional cortical circuits influences information processing at the cortico-hippocampal level. The long-term synaptic and excitatory alterations in hippocampus that we observed, as a consequence of the induction of synaptic plasticity in the adult primary VC, may provide an opportunity for network reconfiguration across temporally-spaced episodes: a mechanism by which memory formation is believed to be initiated. Our data indicate that synaptic plasticity in the primary VC that occurs as a consequence of active sensory processing may lower the threshold for induction of hippocampal synaptic plasticity and thus comprise one of the endogenous triggers for synaptic plasticity in the hippocampus.

\section{ACKNOWLEDGMENTS}

This research was supported by the German Research Foundation's (DFG) Collaborative Research Center "Neurovision" (SFB 509). We are very grateful to Arthur Bikbaev and Jens Klausnitzer for technical advice and assistance.

Bland, B. H. (1986). The physiology and pharmacology of hippocampal formation theta rhythms. Prog. Neurobiol. 26, 1-54.

Buzsáki, G. (1989). Two-stage model of memory trace formation: a role for "noisy" brain states. Neuroscience 31, 551-570.

Buzsáki, G. (2002). Theta oscillations in the hippocampus. Neuron 33, 325-340.
Buzsáki, G.,Haas, H.L., and Anderson,E.G. (1987).Long-term potentiation induced by physiologically relevant stimulus patterns. Brain Res. 435, 331-333.

Buzsáki, G., Llinás, R., Berthoz, A., and Christen, Y. (1994). Temporal Coding in the Brain. Berlin, Springer-Verlag.

Castro, C. A., Silbert, L. H., McNaughton, B. L., and Barnes, C. A. (1989). Recovery of spatial learning deficits 
after decay of electrically induced synaptic enhancement in the hippocampus. Nature 342, 545-548.

Davis, C. D., Jones, F. L., and Derrick, B.E. (2004). Novel environments enhance the induction and maintenance of long-term potentiation in the dentate gyrus. J. Neurosci. 24, 6497-6506.

Eichenbaum, H. (2000). A corticalhippocampal system for declarative memory. Nat. Rev. Neurosci. 1, 41-50.

Engel, A. K., Fries, P., and Singer, W. (2001). Dynamic predictions: oscillations and synchrony in top-down processing. Nat. Rev. Neurosci. 2, 704-716.

Fell, J., Klaver, P., Lehnertz, K., Grunwald, T., Schaller, C., Elger, C. E., and Fernandez, G. (2001). Human memory formation is accompanied by rhinal-hippocampal coupling and decoupling. Nat. Neurosci. 4, 1159-1160.

Fries, P., Neuenschwander, S., Engel, A. K., Goebel, R., and Singer, W. (2001). Rapid feature selective neuronal synchronization through correlated latency shifting. Nat. Neurosci. 4, 194-200.

Fries, P., Nikolic, D., and Singer, W. (2007). The gamma cycle. Trends Neurosci.30, 309-316.

Gray, C. M. (1994). Synchronous oscillations in neuronal systems: mechanisms and functions. J. Comput. Neurosci. 1, 11-38.

Green, E. J., McNaughton, B. L., and Barnes, C. A. (1990). Explorationdependent modulation of evoked responses in fascia dentata: dissociation of motor, EEG, and sensory factors and evidence for synaptic efficacy change. J. Neurosci. 10, 1455-1471.

Harris, K. D., Henze, D. A., Hirase, H., Leinekugel, X., Dragoi, G., Czurko, A., and Buzsáki, G. (2002). Spike train dynamics predicts theta related phase precession in hippocampal pyramidal cells. Nature 417, 738-741.

Hegde, J. (2006). Search for the neural correlates of learning to discriminate orientations. J. Neurosci. 26 , 8877-8878.

Heynen, A. J., and Bear, M. F. (2001). Long-term potentiation of thalamocortical transmission in the adult visual cortex in vivo. J. Neurosci. 21 , 9801-9813.

Heynen, A. J., Quinlan, E. M., Bae, D. C., and Bear, M. F. (2000). Bidirectional, activity dependent regulation of glutamate receptors in the adult hippocampus in vivo. Neuron 28 , 527-536.

Hughes, H.C. (1977). Anatomical and neurobehavioral investigations concerning the thalamocortical organization of the rat's visual system. J. Comp. Neurol. 175 , 311-336.

Ji, D., and Wilson, M. A. (2007). Coordinated memory replay in the visual cortex and hippocampus during sleep. Nat. Neurosci. 10, 100-107.

Jouvet, M. (1969). Biogenic amines and the states of sleep. Science 163, 32-41.

Kahana, M. J., Sekuler, R., Caplan, J. B., Kirschen, M., and Madsen, J. R. (1999). Human theta oscillations exhibit task dependence during virtual maze navigation. Nature 399 , 781-784.

Karmarkar, U. R., and Dan, Y. (2006) Experience-dependent plasticity in adult visual cortex. Neuron 52 577-585.

Kemp, A., and Manahan-Vaughan, D. (2007). Hippocampal LTD: master or minion in declarative memory processes? Trends Neurosci. 30, 111-118.

Kemp, A., and Manahan-Vaughan, D. (2008). The hippocampal CA1 region and dentate gyrus differentiate between environmental and spatial feature encoding through long-term depression. Cereb. Cortex $18,968-977$

Kithigina, V., Vankov, A., Harley, C., and Sara, S. J. (1997). Novelty-elicited, noradrenaline-dependent enhancement of excitability in the dentate gyrus. Eur. J. Neurosci. 9, 41-47.

Larson, J., and Lynch, G. (1986). Induction of synaptic potentiation in the hippocampus by patterned stimulation involves two events. Science 232, 985-988.

Larson, J., and Lynch, G. (1989). Theta pattern stimulation and the induction of LTP: the sequence in which synapses are stimulated determines the degree to which they potentiate. Brain Res. 489, 49-58.

Larson, J., Wong, D., and Lynch, G. (1986) Patterned stimulation at the theta frequency is optimal for the induction of hippocampal long-term potentiation. Brain Res. 368, 347-350.

Lisman, J. E. (1999). Relating hippocampal circuitry to function: recall of memory sequences by reciprocal dentate/CA3 interactions. Neuron 22 , $233-242$.

Liu, L., Wong, T. P., Pozza, M. F., Lingenhoehl, K., Wang, Y., Sheng, M., Auberson, Y.P., and Wang, Y.T. (2004) Role of NMDA receptor subtypes in governing the direction of hippocampal synaptic plasticity. Science 304 , 1021-1024.

Madriaga, M. A., McPhee, L. C., Chersa, T., Christie, K. J., and Whelan, P. J. (2004). Modulation of locomotor activity by multiple 5-HT and dopaminergic receptor subtypes in the neonatal mouse spinal cord. J. Neurophysiol. 92 , 1566-1576.

Majewska, A. K., Newton, J. R., and Sur, M. (2006). Remodeling of synaptic structure in sensory cortical areas in vivo. J. Neurosci. 26, 3021-3029.

Martinez-Conde, S., Macknik, S. L., and Hubel, D. H. (2002). The function of bursts of spikes during visual fixation in the awake primate lateral geniculate nucleus and primary visual cortex. Proc. Natl. Acad. Sci. U.S.A. 99 13920-13925.

Miyamoto, H., and Hensch, T. K. (2003). Reciprocal interaction of sleep and synaptic plasticity. Mol. Interv. 3 , 404-417.

Molnar, Z., Adams, R., and Blakemore, C (1998). Mechanisms underlying the early establishment of thalamocortical connections in the rat. J. Neurosci. 18 5723-5745.

Morris, R. G. M., and Frey, U. (1997) Hippocampal synaptic plasticity: role in spatial learning or the automatic recording of attended experience? Philos. Trans. R. Soc. Lond., B, Biol. Sci. 352, 1489-1503.

Moser, E. I. (1995). Learning-related changes in hippocampal field potentials. Behav. Brain Res. 71, 11-18.

Moser, E. I., Moser, M. B., and Andersen, P. (1994). Potentiation of dentate synapses initiated by exploratory learning in rats: dissociation from brain temperature, motor activity, and arousal. Learn. Mem. 1, 55-73.

Moser, M. B., and Moser, E. I. (1998) Functional differentiation in the hippocampus. Hippocampus 8, 608-619.

Mouly, A. M., and Gervais, R. (2002) Polysynaptic potentiation at different levels of rat olfactory pathways following learning. Learn. Mem. 9, 66-75.

O'Keefe, J., and Nadel, L. (1978). Hippocampus as a Cognitive Map. Oxford, Clarindon.

O'Keefe, J., and Recce, M. L. (1993). Phase relationship between hippocampal place units and the EEG theta rhythm. Hippocampus 3, 317-330.

Pearlstein, E., Mabrouk, F. B., Pflieger, J.F. and Vinay, L. (2005). Serotonin refines the locomotor-related alternations in the in vitro neonatal rat spinal cord Eur. J. Neurosci. 21, 1338-1346.

Rose, G., and Dunwiddie, T. V. (1986) Induction of hippocampal long-term potentiation using physiologically patterned stimulation. Neurosci. Lett. 69 , 244-248.

Sainsbury, R. S., Harris, J.L., and Rowland, G.L. (1987). Sensitization and hippocampal type 2 theta in the rat. Physiol. Behav. 41, 489-493.

Seidenbecher, T., Laxmi, T. R., Stork, O., and Pape, H. C. (2003). Amygdalar and hippocampal theta rhythm synchronization during fear memory retrieval. Science 301, 846-850.

Sharp, P. E., McNaughton, B. L., and Barnes, C. A. (1989). Explorationdependent modulation of evoked responses in fascia dentata: fundamental observations and time course. Psychobiology 17, 257-269.

Singer, W. (1993). Synchronization of cortical activity and its putative role in information processing and learning. Annu. Rev. Physiol. 55, 349-374.

Singer, W., and Gray, C. M. (1995). Visual feature integration and the temporal correlation hypothesis. Annu. Rev. Neurosci. 18, 555-586.

Sirota, A., Montgomery, S., Fujisawa, S., Isomura, Y., Zugaro, M., and Buzsaki, G. (2008). Entrainment of neocortical neurons and gamma oscillations by the hippocampal theta rhythm. Neuron 60, 683-697.

Skaggs, W.E., McNaughton, B. L., Wilson, M. A., and Barnes, C. A. (1996). Theta phase precession in hippocampal neuronal populations and the compression of temporal sequences. Hippocampus 6, 149-172.

Stanton, P. K., and Sarvey, J. M. (1987) Norepinephrine regulates long-term potentiation of both the population spike and dendritic EPSP in hippocampal dentate gyrus. Brain Res. Bull. $18,115-119$.

Steffenach, H.A., Witter, M., Moser, M. B., and Moser, E. I. (2005). Spatial memory in the rat requires the dorsolateral band of the entorhinal cortex. Neuron $45,301-313$.

Steriade, M. (1999). Coherent oscillation and short-term plasticity in corticothalamic networks. Trends Neurosci. 22, 337-345.

Sur, M., Schummers, J., and Dragoi, V. (2002). Cortical plasticity: time for a change. Curr. Biol. 12, R168-170.

Traub, R.D., Jefferys, J.G., and Whittington, M.A. (1999).Fast Oscillations in Cortical Circuits. Cambridge, MA, MIT Press.

Truchet, B., Chaillan, F. A., SoumireuMourat, B., and Roman, F. S. (2002). Early integrative processes physiologically observed in dentate gyrus during an olfactory associative training in rat. J. Integr. Neurosci. 1, 101-115.

Tsanov, M., and Manahan-Vaughan, D. (2007a). The adult visual cortex expresses dynamic synaptic plasticity that is driven by the light-dark cycle J. Neurosci. 27, 8414-8421.

Tsanov, M., and Manahan-Vaughan, D. (2007b). Intrinsic, light-independent and visual-activity dependent mechanisms cooperate in the shaping of the field response in rat visual cortex. $J$. Neurosci. 27, 8422-8429.

Tsanov, M., and Manahan-Vaughan, D. (2008). Synaptic plasticity from 
visual cortex to hippocampus: systems integration in spatial information processing. Neuroscientist 14, 584-597.

Tsanov, M., and Manahan-Vaughan, D. (2009). Long-term plasticity is proportional to theta-activity. PLoS ONE 4, e5850. doi: 10.1371/journal. pone. 0005850 .

Vanderwolf, C. H. (1969). Hippocampal electrical activity and voluntary movement in the rat. Electroencephalogr. Clin. Neurophysiol. 26, 407-418.
Varela, F., Lachaux, J. P., Rodriguez, E., and Martinerie, J. (2001). The brainweb: phase synchronization and largescale integration. Nat. Rev. Neurosci. 2, 229-239.

Wespatat, V., Tennigkeit, F., and Singer, W. (2004). Phase sensitivity of synaptic modifications in oscillating cells of rat visual cortex. J. Neurosci. 24, 9067-9075.

Zilles, K. (1985). The Cortex of the Rat. Berlin, Springer.
Conflict of Interest Statement: The authors declare that the research was conducted in the absence of any commercial or financial relationships that could be construed as a potential conflict of interest.

Received: 25 September 2009; paper pending published: 09 October 2009; accepted: 05 November 2009; published online: 23 November 2009.

Citation: Tsanov $M$ and ManahanVaughan D (2009) Visual cortex plasticity evokes excitatory alterations in the hippocampus. Front. Integr. Neurosci. 3:32. doi: 10.3389/neuro.07.032.2009

Copyright (C) 2009 Tsanov and ManahanVaughan. This is an open-access article subject to an exclusive license agreement between the authors and the Frontiers Research Foundation, which permits unrestricted use, distribution, and reproduction in any medium, provided the original authors and source are credited. 